\title{
Value of Capillary Glucose Profiles in Assessing Risk of Nocturnal Hypoglycemia in Type 1 Diabetes Based on Continuous Glucose Monitoring
}

\author{
Qing Ling · Jing Lu $\cdot$ Xiang Li $\cdot$ Chengcheng Qiao $\cdot$ Dalong Zhu $\cdot$ Yan Bi
}

Received: January 14, 2020 / Published online: March 2, 2020

(C) The Author(s) 2020

\section{ABSTRACT}

Introduction: This study aimed to evaluate the occurrence of nocturnal hypoglycemia in type 1 diabetes (T1D) based on continuous glucose monitoring (CGM), and to explore the value of capillary glucose profiles in assessing the risk of nocturnal hypoglycemia. The study also intended to develop a predictive model to identify people with high risk of nocturnal hypoglycemia.

Methods: A total of 169 participants with T1D received 3 days of blinded CGM; meanwhile, their self-monitoring blood glucose (SMBG) profiles were recorded. Logistic regression

Qing Ling and Jing Lu contributed equally to the manuscript.

Enhanced Digital Features To view enhanced digital features for this article go to https://doi.org/10.6084/ m9.figshare.11808513.

Q. Ling · J. Lu · C. Qiao · D. Zhu ( $₫) \cdot$ Y. Bi $(\bowtie)$ Department of Endocrinology, Drum Tower Hospital Affiliated to Nanjing University Medical School, Nanjing, China

e-mail: zhudalong@nju.edu.cn

Y. Bi

e-mail: biyan@nju.edu.cn

X. Li

Department of Endocrinology, Nanjing Drum

Tower Hospital, Peking Union Medical College and Chinese Academy of Medical Sciences, Nanjing, China analyses were used to evaluate contributory factors of nocturnal hypoglycemia. Potential indicators were estimated using area under receiver operator curve (AUC) analyses.

Results: During the retrospective CGM period, $95(56.2 \%)$ participants with T1D reported 238 events of hypoglycemia, and 69 (29.0\%) of these episodes occurred during the nighttime. Increased risk of nocturnal hypoglycemia correlated with lower HbA1c, glycated albumin, and mean blood glucose $(\mathrm{OR}=0.790,0.940$, 0.651 , respectively; $P<0.05$ ) and higher standard deviation, mean amplitude of glycemic excursions, and low blood glucose index $(\mathrm{OR}=$ $1.463,1.168,4.035$, respectively; $P<0.05$ ) after adjustment for age and duration. Of the daily SMBG profiles, fasting blood glucose $(\mathrm{OR}=$ $0.643, P=0.001)$ and blood glucose at bedtime $(\mathrm{OR}=0.851, P=0.037)$ were associated with the occurrence of nocturnal hypoglycemia. The BGn model, which was derived from the variation of capillary glucose, could discriminate individuals with increased risk of nocturnal hypoglycemia $(\mathrm{AUC}=0.774)$.

Conclusions: Nocturnal hypoglycemia constitutes nearly one-third of hypoglycemic events in people with T1D. Strict glycemic control and great fluctuation of glucose are potential contributory factors. Daily SMBG profiles and the BGn model could help assess the risk of nocturnal hypoglycemia in T1D, which may support further development of preventive strategies. 
Keywords: Nocturnal hypoglycemia; Selfmonitoring blood glucose; Type 1 diabetes

\section{Key Summary Points}

Why carry out this study?

Nocturnal hypoglycemia frequently occurs in people with type 1 diabetes (T1D) and causes great harm.

Capillary blood glucose testing remains the fundamental way of self-monitoring in the clinic.

How can we make full use of selfmonitoring profiles to evaluate and prevent nocturnal hypoglycemia in T1D?

\section{What was learned from the study?}

Nocturnal hypoglycemia constituted nearly one-third of hypoglycemic events in people with T1D based on continuous glucose monitoring.

Fasting blood glucose below $6.2 \mathrm{mmol} / \mathrm{L}$ or blood glucose at bedtime below $6.8 \mathrm{mmol} / \mathrm{L}$ indicates a high risk of hypoglycemia during the nighttime.

The BGn model derived from variation of capillary glucose during the daytime presented a high specificity to discriminate patients with increased risk of nocturnal hypoglycemia.

\section{INTRODUCTION}

Hypoglycemia is a frequent and feared complication in the management of type 1 diabetes (T1D). In the global HAT study, self-reported prevalence of hypoglycemia is $83 \%$ in people with T1D, much higher than that in insulintreated people with type 2 diabetes [1]. Nocturnal hypoglycemia was reported in $40.6 \%$ of people with T1D [1]. The Diabetes Control and Complications Trial (DCCT) revealed that around $50 \%$ of severe hypoglycemia episodes were observed during the nighttime $[2,3]$.

Hypoglycemia has a profound influence on a patient's daily behavior, mental health, cardiovascular system, and central nervous system, resulting in an increased risk of cardiac morbidity and mortality [4-7]. Nocturnal hypoglycemia, which is difficult to recognize and may persist for a long time, causes more harm to people with diabetes. Repeated or severe nocturnal hypoglycemia can cause fatal arrhythmia during sleep, leading to the "dead in bed" syndrome [8]. Therefore, nocturnal hypoglycemia needs to be addressed and resolved for T1D.

Blood glucose monitoring is essential to assess whether glycemic targets are being safely achieved [4]. The improvement of monitoring instruments, such as continuous glucose monitoring (CGM) providing detailed information about current glucose levels and trends, is beneficial for the detection and prevention of hypoglycemia $[9,10]$. Nevertheless, CGM is still underutilized in the real world, with around 3\% in the young and $14 \%$ in older patients [11], probably because of low reimbursement rates and technical complexity. Capillary blood glucose testing, on the other hand, remains the fundamental method of self-monitoring of blood glucose (SMBG) in real-word clinical practice. To date, few studies have examined how the variation of glucose based on SMBG may affect the glucose levels during the nighttime. Figuring out the patterns of glucose change based on SMBG profiles may provide direct and practicable information to prevent hypoglycemia.

In this study, the occurrence of hypoglycemia in people with T1D was investigated according to CGM records. The effect of glucose levels and variation on the occurrence of nocturnal hypoglycemia were examined. Further, we explored whether it was possible to assess the risk of nocturnal hypoglycemia based on capillary glucose profiles during the daytime, which would be helpful for safe glycemic control overnight. Additionally, we intended to develop a predictive model to identify people who were likely to experience nocturnal hypoglycemia. 


\section{METHODS}

\section{Ethics Statement}

The study was approved by the Ethics Committee of Drum Tower Hospital Affiliated to Nanjing University Medical School (2019-00702). This was an observational study and the investigators got permission to obtain the anonymous data; thus, written informed consent was not required from each participant. This study was performed in accordance with the Helsinki Declaration of 1964 and its later amendments.

\section{Study Design and Participants}

The study was observational in design, and the data were retrospectively analyzed. A total of 169 people with T1D were included who attended the inpatient clinic of Nanjing Drum Tower Hospital during 2013-2019. Normally, inpatients in our department would be offered CGM when the disease condition and treatment regimen were stable. Individuals were eligible for inclusion if they received CGM for at least $72 \mathrm{~h}$ along with seven capillary glucose tests recorded each day. Exclusion criteria were acute complications of diabetes, severe infection or other stress statuses, history of alcohol intake, and use of oral drugs influencing glucose metabolism.

\section{Clinical Investigations and Laboratory Determinations}

Clinical characteristics were collected including gender, age, blood pressure, BMI, chronic complications associated with diabetes, and doses of insulin injection. Clinical laboratory measurements included HbA1c, C-peptide levels, urine microalbumin to creatinine ratio (UACR), and liver and renal function.

All the participants were monitored for 72 consecutive hours with a CGM instrument (iPro $^{\mathrm{TM}_{2}}$ Professional CGM, Medtronic). Realtime data were masked to the participants and retrospectively analyzed by the clinician. Sensors were inserted subcutaneously and calibrated according to the standard recommendations of the manufacturers. During the period of CGM, capillary glucose was tested (COUNTER TS, Ascensia Diabetes Care Inc.) seven times every day, including blood glucose before and after three meals as well as at bedtime.

Raw data from CGM records were uploaded using CareLink Therapy Management Soft-ware (Medtronic). Indices including standard deviation (SD), the largest amplitude of glucose excursions (LAGE), mean amplitude of glycemic excursions (MAGE), low blood glucose index (LBGI) as well as mean blood glucose (MBG), and mean absolute glucose (MAG) were calculated to estimate blood glucose variability.

\section{Classification of Hypoglycemia}

Hypoglycemia alert value (level 1) is considered as blood glucose of $3.9 \mathrm{mmol} / \mathrm{L}(70 \mathrm{mg} / \mathrm{dL})$ or below, and clinically significant hypoglycemia (level 2) as blood glucose below $3.0 \mathrm{mmol} / \mathrm{L}$ $(54 \mathrm{mg} / \mathrm{dL}$ ). Severe hypoglycemia (level 3) is defined as severe cognitive impairment requiring assistance from another person or recovery. In this study, episodes of hypoglycemia based on CGM reports were defined as blood glucose of $3.9 \mathrm{mmol} / \mathrm{L}(70 \mathrm{mg} / \mathrm{dL})$ or below for more than 20 min with a recovery interval of at least $30 \mathrm{~min}$. If the glucose values of SMBG were inconsistent with CGM at the same time point, we referred to the former for diagnosis of hypoglycemia. Nocturnal hypoglycemia was defined as hypoglycemia occurring between 12 p.m. and 6 a.m.

\section{Statistical Analysis}

Statistical evaluation was performed by SPSS 23.0. Descriptive data were reported as mean $\pm \mathrm{SD}$ where normally distributed or as median (IQR) where skewed. Student $t$ test or the nonparametric Mann-Whitney $U$ test was used to compare data where appropriate. Binary logistic regression analysis was used to disclose the contributory factors of nocturnal hypoglycemia by the LR method. Models revealing glucose variability and potential glucose values 
at night were established on the basis of SMBG data. One patient was not included in the BGn model because of missing data of blood glucose at bedtime preceding nocturnal hypoglycemia. Eight patients were not included in the $\Delta B G$ model because of missing records of blood glucose after dinner or at bedtime. The discriminatory ability of the models was evaluated by receiver operating characteristic curves (ROC) analysis and the area under the ROC curve (AUC). $P$ values less than 0.05 were considered to indicate statistical significance.

\section{RESULTS}

\section{Participants Characteristics}

A total of 169 people with T1D were included in the current study, including 77 male and 92 female participants. The mean age of all the participants was $35.0 \pm 17.2$ years, between 6 and 72 years. Diabetes duration ranged from 0 to 30 years with an average duration of 6.3 years. According to CGM and SMBG reports, participants were further divided into a nonhypoglycemia group $(n=74)$ and a nocturnal hypoglycemia group $(n=45)$ for further analyses. Demographic characteristics of participants, treatment categories, and laboratory data in the two groups are shown in Table 1.

\section{Reporting of Hypoglycemia Based on CGM}

During the retrospective CGM period, 95 of 169 (56.2\%) participants developed hypoglycemia at least once, and 36 participants (21.3\%) experienced hypoglycemia three or more times. Besides, nocturnal hypoglycemia occurred in 45 $(47.4 \%)$ of the participants with hypoglycemia. Overall, 238 hypoglycemic episodes were reported; of these episodes, 200 episodes were level 1, 38 were level 2, and none was severe hypoglycemia.

Most of the hypoglycemia episodes occurred during the nighttime (29.0\%), followed by before lunch $(19.3 \%)$, before dinner $(15.1 \%)$, after lunch $(13.4 \%)$, at bedtime $(10.5 \%)$, after dinner (8.4\%), after breakfast (3.8\%), and fasting (0.4\%) (Fig. 1). The mean level of blood glucose during each hypoglycemic period was $3.3-3.5 \mathrm{mmol} / \mathrm{L}$ with the lowest level of $2.2 \mathrm{mmol} / \mathrm{L}$.

\section{Contributory Factors of Nocturnal Hypoglycemia}

Compared with the non-hypoglycemia group, patients in the nocturnal hypoglycemia group showed significantly lower levels of HbA1c, glycated albumin, and MBG; meanwhile levels of SD, MAGE, and LBGI were elevated in this group (Table 1). Other factors including age, BMI, blood pressure, disease duration, insulin injection strategies, combination of metformin or acarbose, and LAGE were not significantly different between the two groups. Logistic regression analyses revealed that increased risk of nocturnal hypoglycemia was correlated with lower HbA1c levels $(\mathrm{OR}=0.790,95 \%$ CI 0.657 , $0.951 ; P<0.05)$, glycated albumin $(\mathrm{OR}=0.940$, $95 \%$ CI $0.889,0.994 ; P<0.05)$, and MBG $(\mathrm{OR}=0.651,95 \%$ CI $0.523,0.811 ; P<0.001)$ and higher SD $(\mathrm{OR}=1.463,95 \%$ CI 1.007 , 2.128; $P<0.05)$, MAGE $(\mathrm{OR}=1.168,95 \% \mathrm{CI}$ $1.033,1.360 ; P<0.05)$, and LBGI $(\mathrm{OR}=4.035$, $95 \%$ CI 2.290, 7.109; $P<0.001)$ after adjustment for age and duration. Thus, low blood glucose levels and large glycemic fluctuation were potential contributory factors for the development of nocturnal hypoglycemia.

\section{Assessing the Risk of Nocturnal Hypoglycemia Based on Daily Capillary Blood Glucose}

To examine whether the occurrence of hypoglycemia during the nighttime correlated with levels of capillary glucose during the daytime, glucose profiles of SMBG were compared between patients with nocturnal hypoglycemia and those without hypoglycemia. The nocturnal hypoglycemia group showed significantly lower levels of blood glucose before three meals, after dinner, and at bedtime (Table 2). After adjustment for age, duration, HbA1c and MBG, multiple logistic regression analyses showed that the risk of nocturnal hypoglycemia 
Table 1 Demographic, clinical, and laboratory characteristics of the participants enrolled in two groups

\begin{tabular}{|c|c|c|c|}
\hline & Non-hypoglycemia & Nocturnal hypoglycemia & $P$ value \\
\hline Number of participants & 74 & 45 & \\
\hline \multicolumn{4}{|l|}{ Clinical characteristics } \\
\hline Gender $(\mathrm{F} / \mathrm{M})$ & $34 / 40$ & $29 / 16$ & 0.038 \\
\hline Age (years) & $35.7 \pm 17.9$ & $35.6 \pm 17.2$ & 0.989 \\
\hline BMI $\left(\mathrm{kg} / \mathrm{m}^{2}\right)$ & $21.2 \pm 3.6$ & $20.7 \pm 2.9$ & 0.506 \\
\hline Duration of diabetes (years) & $4.0(0.5,10.3)$ & $3.0(1.0,11.0)$ & $0.744^{\dagger}$ \\
\hline Systolic blood pressure $(\mathrm{mmHg})$ & $119.2 \pm 16.1$ & $123.0 \pm 13.8$ & 0.193 \\
\hline Diastolic blood pressure $(\mathrm{mmHg})$ & $72.8 \pm 11.3$ & $75.6 \pm 10.9$ & 0.185 \\
\hline \multicolumn{4}{|l|}{ Laboratory measurements } \\
\hline HbAlc (\%) & $9.1 \pm 2.5$ & $7.9 \pm 2.3$ & 0.009 \\
\hline Glycated albumin (\%) & $26.7 \pm 9.4$ & $22.3 \pm 8.4$ & 0.024 \\
\hline Fasting C-peptide (pmol/L) & $87.9(0,229.0)$ & $53.6(0.0,267.2)$ & $0.955^{\dagger}$ \\
\hline 2-h post-meal C-peptide (pmol/L) & $162.2(5.2,427.3)$ & $128.1(0.0463 .3)$ & $0.696 \dagger$ \\
\hline Alanine transaminase $(\mathrm{U} / \mathrm{L})$ & $23.6 \pm 34.9$ & $156.3 \pm 912.9$ & 0.352 \\
\hline Aspartate transaminase $(\mathrm{U} / \mathrm{L})$ & $20.7 \pm 13.6$ & $18.4 \pm 5.4$ & 0.308 \\
\hline Blood urea nitrogen $(\mathrm{mmol} / \mathrm{L})$ & $5.0 \pm 1.2$ & $4.9 \pm 1.2$ & 0.516 \\
\hline Serum creatinine $(\mu \mathrm{mol} / \mathrm{L})$ & $51.8 \pm 14.8$ & $53.0 \pm 14.4$ & 0.696 \\
\hline Uric acid $(\mathrm{mmol} / \mathrm{L})$ & $274.9 \pm 87.2$ & $269.9 \pm 94.2$ & 0.779 \\
\hline Urine microalbumin to creatinine ratio $(\mathrm{mg} / \mathrm{g})$ & $16.8(7.8,28.3)$ & $16.0(8.4,26.9)$ & $0.563^{\dagger}$ \\
\hline 24-h urine albumin (mg) & $102.0(69.4,161.5)$ & $112.0(77.0,164.0)$ & $0.388^{\dagger}$ \\
\hline Triglycerides $(\mathrm{mmol} / \mathrm{L})$ & $1.1 \pm 1.9$ & $0.8 \pm 0.3$ & 0.213 \\
\hline Total cholesterol $(\mathrm{mmol} / \mathrm{L})$ & $4.3 \pm 1.2$ & $4.2 \pm 0.8$ & 0.611 \\
\hline HDL cholesterol $(\mathrm{mmol} / \mathrm{L})$ & $1.5 \pm 0.5$ & $1.6 \pm 0.5$ & 0.623 \\
\hline LDL cholesterol $(\mathrm{mmol} / \mathrm{L})$ & $2.3 \pm 1.0$ & $2.3 \pm 0.6$ & 0.792 \\
\hline \multicolumn{4}{|l|}{ Treatment } \\
\hline CSII/MDI & $22 / 46$ & $6 / 37$ & 0.143 \\
\hline Metformin $(n)$ & 19 & 11 & 0.276 \\
\hline Acarbose $(n)$ & 11 & 4 & 0.099 \\
\hline \multicolumn{4}{|l|}{ Daily insulin dose } \\
\hline Total daily insulin dose $(\mathrm{U} / \mathrm{kg})$ & $0.48 \pm 0.23$ & $0.49 \pm 0.19$ & 0.849 \\
\hline Daily basal insulin dose $(\mathrm{U} / \mathrm{kg})$ & $0.20 \pm 0.10$ & $0.20 \pm 0.10$ & 0.752 \\
\hline Daily bolus insulin dose $(\mathrm{U} / \mathrm{kg})$ & $0.29 \pm 0.15$ & $0.29 \pm 0.13$ & 0.777 \\
\hline
\end{tabular}


Table 1 continued

\begin{tabular}{lllc}
\hline & Non-hypoglycemia & Nocturnal hypoglycemia & P value \\
\hline $\begin{array}{l}\text { Diabetic complications } \\
\text { With/without }\end{array}$ & $32 / 42$ & & \\
CGM parameters & & $16 / 29$ & 0.263 \\
MBG (mmol/L) & $9.6 \pm 2.2$ & $7.9 \pm 1.8$ & $<0.001$ \\
SD (mmol/L) & $2.3 \pm 1.0$ & $2.5 \pm 1.1$ & 0.043 \\
LAGE (mmol/L) & $9.2 \pm 3.9$ & $10.4 \pm 3.6$ & 0.101 \\
MAGE (mmol/L) & $5.2 \pm 2.2$ & $6.2 \pm 2.8$ & 0.042 \\
LBGI (mmol/L) & $0.7 \pm 1.0$ & $4.3 \pm 3.7$ & $<0.001$ \\
MAG (mmol/L) & $1.7 \pm 0.6$ & $1.9 \pm 0.7$ & 0.158 \\
\hline D. & &
\end{tabular}

Data are presented as mean \pm SD or median (IQR)

$H D L$ cholesterol, high-density lipoprotein cholesterol, $L D L$ cholesterol, low-density lipoprotein cholesterol, CSII continuous subcutaneous insulin infusion, $M D I$ multiple daily insulin injections, $M B G$ mean blood glucose, $S D$ standard deviation, $L A G E$ the largest amplitude of glucose excursions, $M A G E$ mean amplitude of glycemic excursions, $L B G I$ low blood glucose index, $M A G$ mean absolute glucose

$t$ test was used for comparisons between the nocturnal hypoglycemia and non-hypoglycemia group

$\dagger$ Nonparametric Mann-Whitney $U$ test was used for comparison of skewed data

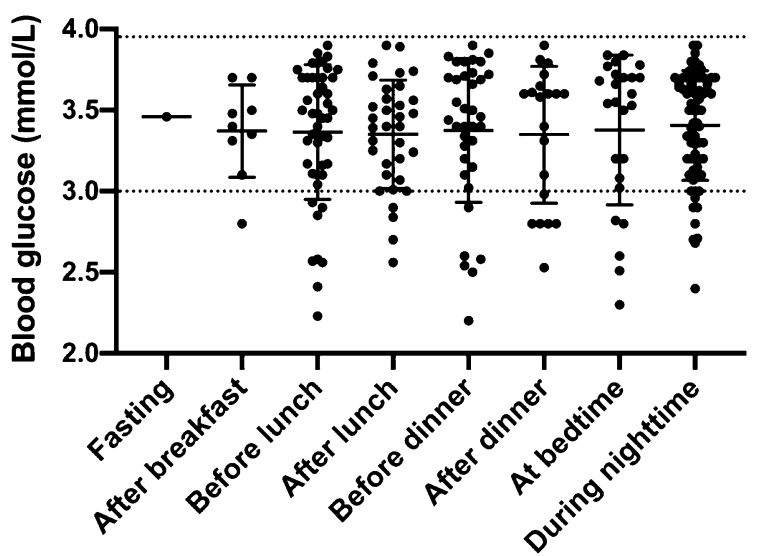

Fig. 1 Occurrence of hypoglycemic events during different time periods based on CGM. Fasting (6:00- 7:00 a.m.), after breakfast (7:30-9:00 a.m.), before lunch (9:00-11:00 a.m.), after lunch (11:30 a.m.- 13:30 p.m.), before dinner (13:30-17:00 p.m.), after dinner (17:30-19:30 p.m.), at bedtime (19:30-24:00 p.m.), during the nighttime (24:00 p.m.-6:00 a.m.). Each dot represents an individual negatively correlated with blood glucose preceding bedtime $(\mathrm{OR}=0.851,95 \%$ CI 0.732, 0.990; $P<0.05)$, as well as with fasting blood glucose the next day $(\mathrm{OR}=0.643,95 \%$ CI $0.491,0.843 ; P<0.05)$.

ROC analyses were performed to further investigate the cutoff values for discrimination of individuals with nocturnal hypoglycemia. Blood glucose at bedtime less than $6.8 \mathrm{mmol} / \mathrm{L}$ might indicate an occurrence of hypoglycemia during the nighttime with a sensitivity of $51.1 \%$ and specificity of $87.3 \%$ (AUC $0.719, P<0.05$ ); the cutoff value for fasting blood glucose was less than $6.2 \mathrm{mmol} / \mathrm{L}$ (sensitivity $77.8 \%$, specificity $72.2 \%$, AUC 0.814, $P<0.01$ ) (Table 3).

\section{Assessing the Risk of Nocturnal Hypoglycemia Based on Models Derived from SMBG}

Participants in this study were all inpatients offered relatively fixed lifestyle intervention, and CGM was generally prescribed when they 
Table 2 Comparison of capillary blood glucose profiles between non-hypoglycemia and nocturnal hypoglycemia group

\begin{tabular}{lcrr}
\hline & Non-hypoglycemia & Nocturnal hypoglycemia & P value \\
\hline Day preceding nocturnal hypoglycemia & & \\
Fasting & $8.6 \pm 3.3$ & $6.4 \pm 2.7$ & $<0.001$ \\
After breakfast & $12.5 \pm 4.5$ & $10.6 \pm 5.3$ & 0.053 \\
Before lunch & $10.8 \pm 4.3$ & $8.2 \pm 4.7$ & 0.005 \\
After lunch & $9.5 \pm 3.4$ & $8.4 \pm 4.0$ & 0.153 \\
Before dinner & $9.7 \pm 2.9$ & $7.7 \pm 2.8$ & 0.001 \\
After dinner & $10.4 \pm 4.3$ & $9.0 \pm 2.8$ & 0.037 \\
At bedtime & $10.3 \pm 3.6$ & $7.7 \pm 3.1$ & $<0.001$ \\
Day of nocturnal hypoglycemia & & & $<0.001$ \\
Fasting & $7.9 \pm 3.1$ & $5.4 \pm 2.1$ & 0.548 \\
After breakfast & $11.3 \pm 4.1$ & $10.8 \pm 3.9$ &
\end{tabular}

Data are presented as mean \pm SD

$t$ test was used for comparisons between the nocturnal hypoglycemia and non-hypoglycemia group

Data of participants in the non-hypoglycemia group were retrieved and calculated on the basis of the second and third day of CGM records

Table 3 ROC analyses of fasting blood glucose, blood glucose at bedtime, BGn model, and $\Delta \mathrm{BG}$ model

\begin{tabular}{lcllll}
\hline & Cutoff value & Sensitivity (\%) & Specificity (\%) & Youden's index & AUC \\
\hline Fasting BG & 6.2 & 77.8 & 72.2 & 0.5 & 0.814 \\
BG at bedtime & 6.8 & 51.1 & 87.3 & 0.38 & 0.719 \\
BGn & 5.3 & 51.1 & 94.5 & 0.46 & 0.774 \\
$\Delta$ BG & -1.2 & 67.4 & 66.2 & 0.34 & 0.646 \\
\hline
\end{tabular}

$B G$ blood glucose, $A U C$ area under receiver operator curve

$\mathrm{BGn}=\mathrm{BG}$ at bedtime $\times\left(1-\mathrm{SD}_{\mathrm{SM}} / \mathrm{MBG}_{\mathrm{SM}}\right) ; \Delta \mathrm{BG}=\mathrm{BG}$ at bedtime $-\mathrm{BG}$ after dinner

were in a stable condition. Therefore, glucose variability was mainly influenced by patients' intrinsic factors and pharmacokinetics of insulin. Next, we tried to figure out whether the risk of nocturnal hypoglycemia could be indicated by the variability of capillary glucose during the daytime.

Based on SMBG profiles, two models were established to evaluate the risk of nocturnal hypoglycemia: (1) $\Delta \mathrm{BG}=$ blood glucose at bedtime - blood glucose after dinner. This model revealed a decrease in blood glucose from post- dinner to bedtime. (2) BGn = blood glucose at bedtime $\times\left(1-\mathrm{SD}_{\mathrm{SM}} / \mathrm{MBG}_{\mathrm{SM}}\right) . \quad \mathrm{SD}_{\mathrm{SM}}$ and $\mathrm{MBG}_{\mathrm{SM}}$ represented the standard deviation and the mean glucose levels before three meals and at bedtime just before the occurrence of nocturnal hypoglycemia. This model revealed the predicted blood glucose at night according to the variation of daily blood glucose. For the non-hypoglycemia group, $\Delta \mathrm{BG}$ and $\mathrm{BGn}$ were calculated using the average values of capillary glucose during the last two days of the CGM period. 

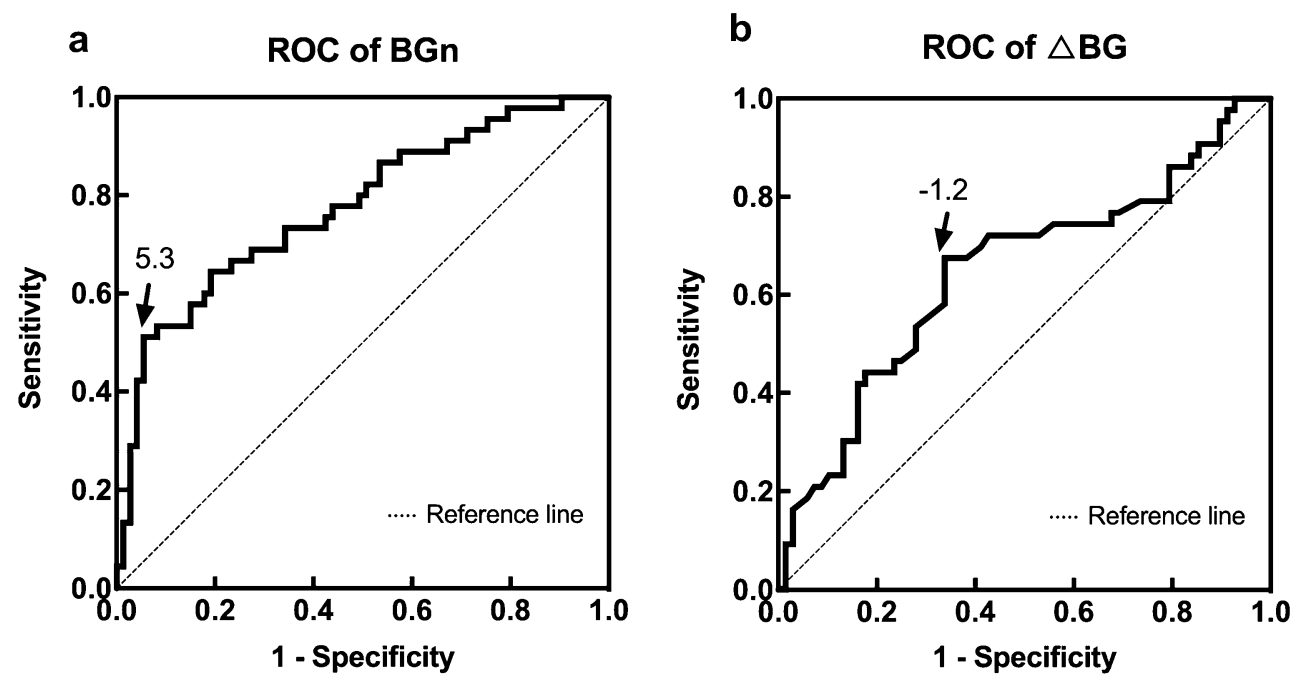

Fig. 2 ROC of BGn model (a) and $\triangle B G$ model (b) in assessing risk of nocturnal hypoglycemia among people with T1D

Patients in the nocturnal hypoglycemia group showed significantly greater $\Delta \mathrm{BG}[-1.5$ $(-2.5,0.3)$ vs $-0.6(-1.7,0.7), P<0.05]$ and lower BGn $(5.5 \pm 2.1$ vs $7.7 \pm 2.2$ vs, $P<0.001$ ) than those in the non-hypoglycemia group. Increased odds of nocturnal hypoglycemia were associated with lower BGn $(\mathrm{OR}=0.588,95 \%$ CI $0.464,0.746 ; P<0.001)$ and greater $\triangle \mathrm{BG}(\mathrm{OR}=0.828,95 \%$ CI 0.696 , $0.986 ; P=0.034)$. After adjustment for age, disease duration, HbA1c and MBG levels, the BGn model could discriminate individuals with increased risk of nocturnal hypoglycemia $\left(\right.$ AUC $\left._{\mathrm{ROC}} 0.774, P<0.001\right)$ with a cutoff point of $5.2 \mathrm{mmol} / \mathrm{L}$ (sensitivity $51.1 \%$, specificity $94.5 \%$ ). The AUC of $\triangle \mathrm{BG}$ model was 0.646 , and the cutoff point was $-1.2 \mathrm{mmol} / \mathrm{L}$ (sensitivity 67.4\%, specificity 66.2\%) (Fig. 2, Table 3).

\section{DISCUSSION}

Hypoglycemia is a common acute complication in people with $\mathrm{T} 1 \mathrm{D}$ and can pose a constant danger to them and their families [4]. In our study, 95 of 169 (56.2\%) participants reported hypoglycemia at least once during 3 days of CGM. Most episodes of hypoglycemia occurred during the nighttime, which was similar to our previous report in type 2 diabetes [12]. The increase in nocturnal hypoglycemia may be due to an attenuated awareness of discomfort and rarely performed self-monitoring of blood glucose [13]. Therefore, detection and prevention of nocturnal hypoglycemia should be especially addressed in people with T1D.

In the current study, low levels of HbA1c and glycated albumin were significantly associated with the occurrence of nocturnal hypoglycemia, indicating strict glycemic control as a potential risk factor for nocturnal hypoglycemia. This was supported by the DCCT studies, in which the intensive group was three times more likely to develop severe hypoglycemia [2, 3]. During the Epidemiology of Diabetes Interventions and Complications (EDIC) study, the risk of severe hypoglycemia increased by $13 \%-15 \%$ for a $10 \%$ lower HbA1c [14]. Nevertheless, another study observed a J-shaped relationship between HbA1c and hypoglycemia in T1D, suggesting that a high level of blood glucose also increased the risk of hypoglycemia [15]. Considering the heterogeneity in clinical characteristics and disease progression of T1D, targets of glycemic control should be individualized to ensure safely controlled diabetes.

Glycemic variability provides more information than the mean level of glucose for the evaluation of hypoglycemic risk $[16,17,18]$. In our study, CGM indices of glycemic fluctuation including SD, MAGE, and LBGI were positively correlated with the risk of nocturnal hypoglycemia. Many studies focused on predicting hypoglycemia or hyperglycemia via indices or 
algorithms based on CGM data $[19,20,21]$, which is fundamental for development of closed-loop insulin pump therapy. However, the application of these advanced technologies in the clinic is still limited worldwide. Capillary blood glucose monitoring remains widely used because of its convenience, accuracy, and low cost. SMBG via capillary testing is an important approach in lifestyle self-education for people with T1D. Besides, SMBG at some time points or glycemic tendency according to daily profiles may imply hypo- or hyperglycemia during the next few hours, especially when lifestyle and insulin therapy maintained stable.

In this study, we identified that fasting glucose and blood glucose at bedtime were good evaluators of nocturnal hypoglycemia risk even after adjustment for multiple variables. The ROC analysis-derived cutoff value of fasting blood glucose was $6.2 \mathrm{mmol} / \mathrm{L}$, with a sensitivity of $77.8 \%$ and specificity of $72.2 \%$. A similar study reported a cutoff value of fasting glucose of $7.5 \mathrm{mmol} / \mathrm{L}$ [22]. The inconsistent cutoff values reported may due to the different study populations, sample sizes, and lifestyles. Moreover, using Youden's index statistic, we discovered that blood glucose at bedtime of less than $6.8 \mathrm{mmol} / \mathrm{L}$ acted as a warning alarm for high risk of nocturnal hypoglycemia in people with T1D (sensitivity $51.1 \%$ and specificity $87.3 \%$ ). This cutoff value was consistent with the glycemic target recommendations in the international guidelines [23, 24].

Instead of time point capillary glucose, daily SMBG profiles provide more information about the variability of blood glucose. Mathioudakis et al. established a prediction model for insulinassociated hypoglycemia in non-critically ill hospitalized adults. The model is based on multiple variables including mean BG, nadir BG, BG coefficient of variation, age, weight, diet status, insulin doses, types of diabetes, stage of chronic kidney disease, and liver function [25]. The sensitivity and specificity of this model were $81.9 \%$ and $78.6 \%$. Another study proposed an index $\left(\% \mathrm{CV}_{\mathrm{SMBG}}\right)$ derived from SMBG profiles to discriminate hypoglycemia in 60 people with T1D [26]. A $\% \mathrm{CV}_{\mathrm{SMBG}}$ of over $44 \%$ provided the best discrimination ability with a sensitivity of $81.3 \%$ and specificity of $89 \%$. These studies provided some clues for real-time preventive interventions in insulin-treated individuals, yet neither was specific for the occurrence of nocturnal hypoglycemia in T1D.

In the current study, the BGn model showed a discriminatory ability (AUC $=0.774$ ) for individuals with an increased risk of nocturnal hypoglycemia. The specificity reached $94.5 \%$ when $\mathrm{BGn}$ was $5.3 \mathrm{mmol} / \mathrm{L}$. It is more specific than studies mentioned above in estimating the risk of hypoglycemia during the nighttime. Besides, BGn was a simplified model only involving pre-meal and bedtime glucose, which was easier to comprehend and apply. In our study, the model was applied especially to people with T1D and was convenient to make a rapid assessment for the risk of nocturnal hypoglycemia.

There are several limitations in this study. The data were obtained from a population with a relatively small sample size. Analyses and models were made on the basis of data of inpatients; thus, application of our findings may require generally stable lifestyle and insulin regimens. A further prospective study is required to confirm the value of fasting blood glucose, blood glucose at bedtime, and the BGn model for predicting nocturnal hypoglycemia. Another limitation was that CGM measured glucose levels in the interstitial space, which could be inconsistent with capillary blood glucose and cause over-reporting of hypoglycemia.

\section{CONCLUSIONS}

Hypoglycemia frequently occurred during the nighttime in people with T1D. Low HbA1c, reduced mean blood glucose, and great fluctuation of glucose were contributory factors for nocturnal hypoglycemia. Individualized glycemic targets should be emphasized for safe control of diabetes. On the basis of capillary glucose profiles, fasting blood glucose, blood glucose at bedtime, and the BGn model were identified as potential indicators for nocturnal hypoglycemia. Our study addressed the detection and prediction of hypoglycemia during sleep in T1D, which may support further development of preventive strategies. 


\section{ACKNOWLEDGEMENTS}

We thank the participants of the study.

Funding. This study and the journal's Rapid Service Fee were sponsored by grants from the National Key Research and Development Program of China (2017YFC1309605), Nanjing Health Youth Talent (QRX17123) and Jiangsu Provincial Key Medical Discipline (XK201105, ZDXKB2016012).

Authorship. All named authors meet the International Committee of Medical Journal Editors (ICMJE) criteria for authorship for this article, take responsibility for the integrity of the work as a whole, and have given their approval for this version to be published.

Authorship Contributions. Q.L. and J.L. participated in data collection, performed the statistical analysis, prepared figures and tables, and wrote the manuscript. X.L. participated in data collection and the interpretation of data. C.C.Q. participated in data collection. D.L.Z. supervised the project and designed the study. Y.B. supervised the project, designed the study, interpreted the results, and co-wrote the manuscript.

Disclosures. Qing Ling, Jing Lu, Xiang Li, Chengcheng Qiao, Dalong Zhu and Yan Bi declare that they have nothing to disclose.

Compliance with Ethics Guidelines. The study was approved by the Ethics Committee of Drum Tower Hospital Affiliated to Nanjing University Medical School (2019-007-02). This was an observational study and the investigators got permission to obtain the anonymous data; thus, written informed consent was not required from each participant. This study was performed in accordance with the Helsinki Declaration of 1964 and its later amendments.

Data Availability. The datasets generated during and/or analyzed during the current study are available from the corresponding author on reasonable request.
Open Access. This article is licensed under a Creative Commons Attribution-NonCommercial 4.0 International License, which permits any noncommercial use, sharing, adaptation, distribution and reproduction in any medium or format, as long as you give appropriate credit to the original author(s) and the source, provide a link to the Creative Commons licence, and indicate if changes were made. The images or other third party material in this article are included in the article's Creative Commons licence, unless indicated otherwise in a credit line to the material. If material is not included in the article's Creative Commons licence and your intended use is not permitted by statutory regulation or exceeds the permitted use, you will need to obtain permission directly from the copyright holder. To view a copy of this licence, visit http://creativecommons.org/ licenses/by-nc/4.0/.

\section{REFERENCES}

1. Khunti K, Alsifri S, Aronson R, et al. Rates and predictors of hypoglycaemia in 27585 people from 24 countries with insulin-treated type 1 and type 2 diabetes: the global HAT study. Diabetes Obes Metab. 2016;18(9):907-15.

2. The DCCT Research Group. Epidemiology of severe hypoglycemia in the diabetes control and complications trial. Am J Med. 1991;90(4):450-9.

3. Nathan DM, Genuth S, Lachin J, et al. The effect of intensive treatment of diabetes on the development and progression of long-term complications in insulin-dependent diabetes mellitus. N Engl J Med. 1993;329(14):977-86.

4. Frier BM. Hypoglycaemia in diabetes mellitus: epidemiology and clinical implications. Nat Rev Endocrinol. 2014;10(12):711-22.

5. Akirov A, Grossman A, Shochat T, Shimon I. Mortality among hospitalized patients with hypoglycemia: insulin related and noninsulin related. J Clin Endocrinol Metab. 2017;102(2):416-24.

6. Novodvorsky P, Bernjak A, Chow E, et al. Diurnal differences in risk of cardiac arrhythmias during spontaneous hypoglycemia in young people with type 1 diabetes. Diabetes Care. 2017;40(5):655-62.

7. Hsieh YC, Liao YC, Li CH, et al. Hypoglycaemic episodes increase the risk of ventricular arrhythmia 
and sudden cardiac arrest in patients with type 2 diabetes-a nationwide cohort study. Diabetes Metab Res Rev. 2020;36(2):e3226.

8. Tattersall RB, Gill GV. Unexplained deaths of type 1 diabetic patients. Diabet Med. 1991;8(1):49-58.

9. Lind M, Polonsky W, Hirsch IB, et al. Continuous glucose monitoring vs conventional therapy for glycemic control in adults with type 1 diabetes treated with multiple daily insulin injections: the gold randomized clinical trial. JAMA. 2017;317(4): 379-87.

10. Heinemann L, Freckmann G, Ehrmann D, et al. Real-time continuous glucose monitoring in adults with type 1 diabetes and impaired hypoglycaemia awareness or severe hypoglycaemia treated with multiple daily insulin injections (HypoDE): a multicentre, randomised controlled trial. Lancet. 2018;391(10128):1367-77.

11. Beck RW, Tamborlane WV, Bergenstal RM, et al The T1D exchange clinic registry. J Clin Endocrinol Metab. 2012;97(12):4383-9.

12. Bi Y, Yan J, Tang W, Yang J, Weng J. Prevalence of hypoglycemia identified by intensive bedside glucose monitoring among hospitalized patients with diabetes mellitus. J Diabetes. 2013;5(3):300-1.

13. Allen KV, Frier BM. Nocturnal hypoglycemia: clinical manifestations and therapeutic strategies toward prevention. Endocr Pract. 2003;9(6):530-43.

14. Gubitosi-Klug RA, Braffett BH, White NH, et al. Risk of severe hypoglycemia in type 1 diabetes over 30 years of follow-up in the DCCT/EDIC study. Diabetes Care. 2017;40(8):1010-6.

15. Gimenez M, Tannen AJ, Reddy M, et al. Revisiting the relationships between measures of glycemic control and hypoglycemia in continuous glucose monitoring data sets. Diabetes Care. 2018;41(2): 326-32.

16. Qu Y, Jacober SJ, Zhang Q, Wolka LL, DeVries JH. Rate of hypoglycemia in insulin-treated patients with type 2 diabetes can be predicted from glycemic variability data. Diabetes Technol Ther. 2012;14(11):1008-122.

17. Rodbard D. Hypo- and hyperglycemia in relation to the mean, standard deviation, coefficient of variation, and nature of the glucose distribution. Diabetes Technol Ther. 2012;14(10):868-76.

18. Monnier L, Colette C, Wojtusciszyn A, et al. Toward defining the threshold between low and high glucose variability in diabetes. Diabetes Care. 2017;40(7):832-8.

19. Harvey RA, Dassau E, Zisser HC, et al. Clinically relevant hypoglycemia prediction metrics for event mitigation. Diabetes Technol Ther. 2012;14(8): 719-27.

20. Georga EI, Protopappas VC, Ardigo D, Polyzos D, Fotiadis DI. A glucose model based on support vector regression for the prediction of hypoglycemic events under free-living conditions. Diabetes Technol Ther. 2013;15(8):634-43.

21. Tkachenko P, Kriukova G, Aleksandrova M, et al. Prediction of nocturnal hypoglycemia by an aggregation of previously known prediction approaches: proof of concept for clinical application. Comput Methods Programs Biomed. 2016;134:179-86.

22. Mitsuishi S, Nishimura R, Ando K, Tsujino D, Utsunomiya K. Can fasting glucose levels or postbreakfast glucose fluctuations predict the occurrence of nocturnal asymptomatic hypoglycemia in type 1 diabetic patients receiving basal-bolus insulin therapy with long-acting insulin? PLoS One. 2015;10(12):e0144041.

23. American Diabetes Association. 13. Children and adolescents: standards of medical care in diabetes2019. Diabetes Care. 2019;42(Suppl 1):S148-S164.

24. DiMeglio LA, Acerini CL, Codner E, et al. ISPAD Clinical Practice Consensus Guidelines 2018: glycemic control targets and glucose monitoring for children, adolescents, and young adults with diabetes. Pediatr Diabetes. 2018;19(27):105-14.

25. Mathioudakis NN, Everett E, Routh S, et al. Development and validation of a prediction model for insulin-associated hypoglycemia in non-critically ill hospitalized adults. BMJ Open Diabetes Res Care. 2018;6(1):e000499.

26. Rama Chandran S, Wl T, Lye WK, et al. Beyond HbA1c: comparing glycemic variability and glycemic indices in predicting hypoglycemia in type 1 and type 2 diabetes. Diabetes Technol Ther. 2018;20(5):353-62. 